\title{
P-0512- EFFICACY AND CARDIOVASCULAR EFFECTS OF DIPEPTIDYL PEPTIDASE-4 (DPP4) INHIBITORS: A SYSTEMATIC REVIEW.
}

\section{BACKGROUND}

\begin{abstract}
The dipeptidyl peptidase-4 (DPP4) inhibitors (or "gliptins") are an important new class of incretin-based therapy for treatment of type 2 diabetes mellitus (T2DM). These drugs prolong the action of incretin hormones glucagon-like peptide-1 (GLP-1) and glucose-dependent insulinotropic polypeptide (GIP), by inhibiting their breakdown.The incretin hormones increase insulin secretion and suppress glucagon levels. Evidence on the efficacy of these drugs in controlling glycemic levels in T2DM has been accumulating. Furthermore, given that the risk of cardiovascular (CV) disease is substantially greater in people with diabetes compared to those without diabetes, CV safety profiles of these agents have been considered important in their selection for T2DM treatment.
\end{abstract}

We reviewed the efficacy and CV effects of DPP-4 inhibitors from published reports and randomized controlled trials (RCT), e.g. SAVOR-TIMI 53, EXAMINE, and TECOS, for evidence that these drugs are effective in controlling gyclemia and have acceptable CV profiles in adult patients with T2DM.

A total of 59 studies met the selection criteria (Alogliptin 7, Linagliptin 7, Saxagliptin 8, Vildagliptin 15, Sitagliptin 22). The reviewed studies differed with regard to patient population and duration. However, DPP-4 inhibitors conclusively showed efficacy in lowering glycemic levels and achieving a meaningful reduction of $\mathrm{HbA}$ from the baseline when used as a monotherapy or as an add-on therapy, with a majority of T2DM patients achieving HbA1c levels of $<7 \%$. The Saxagliptin 1c SAVOR-TIMI 53 trial showed that the drug is safe for cardiovascular patients with no significant increase in endpoint of MACE (HR1.02, CI 0.94-1.11, p=0.66), but slightly increases the risk of hospitalization with heart failures. EXAMINE trial using Alogliptin also showed the same results with no significant increase in MACE events as compared to placebo (HR 0.96, $\mathrm{p}=<0.001$ ). The TECOS trial using Sitagliptin did not show increased risk for major adverse CV events or hospitalization for heart failure. The differences in heart failure outcomes notwithstanding, the studies show similar findings for hospitalization for unstable angina, myocardial infarction, and CV death..

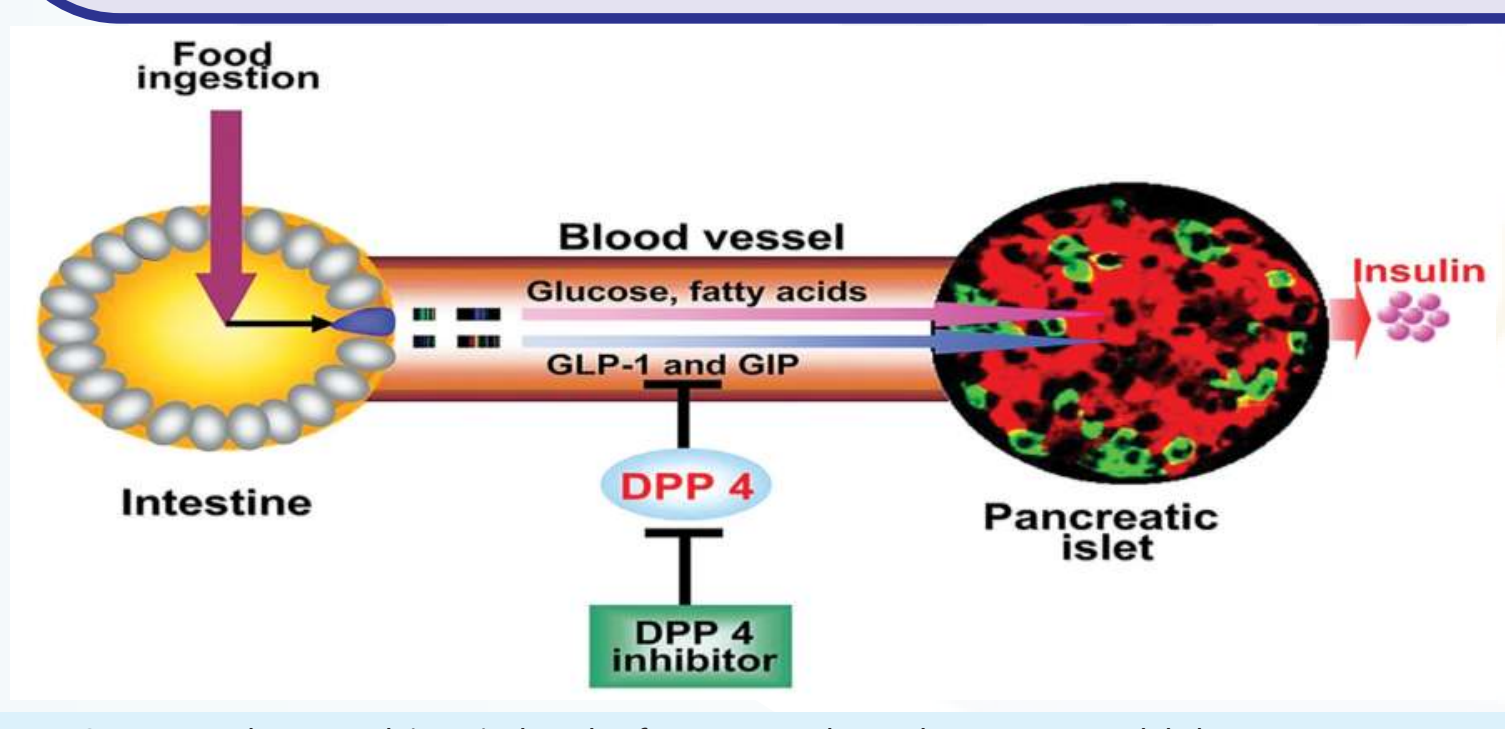

Source: Wook Kim, et al. (2008). The role of incretins in glucose homeostasis and diabetes treatment. Pharmacology reviews, 60(4), pp. 470-512.

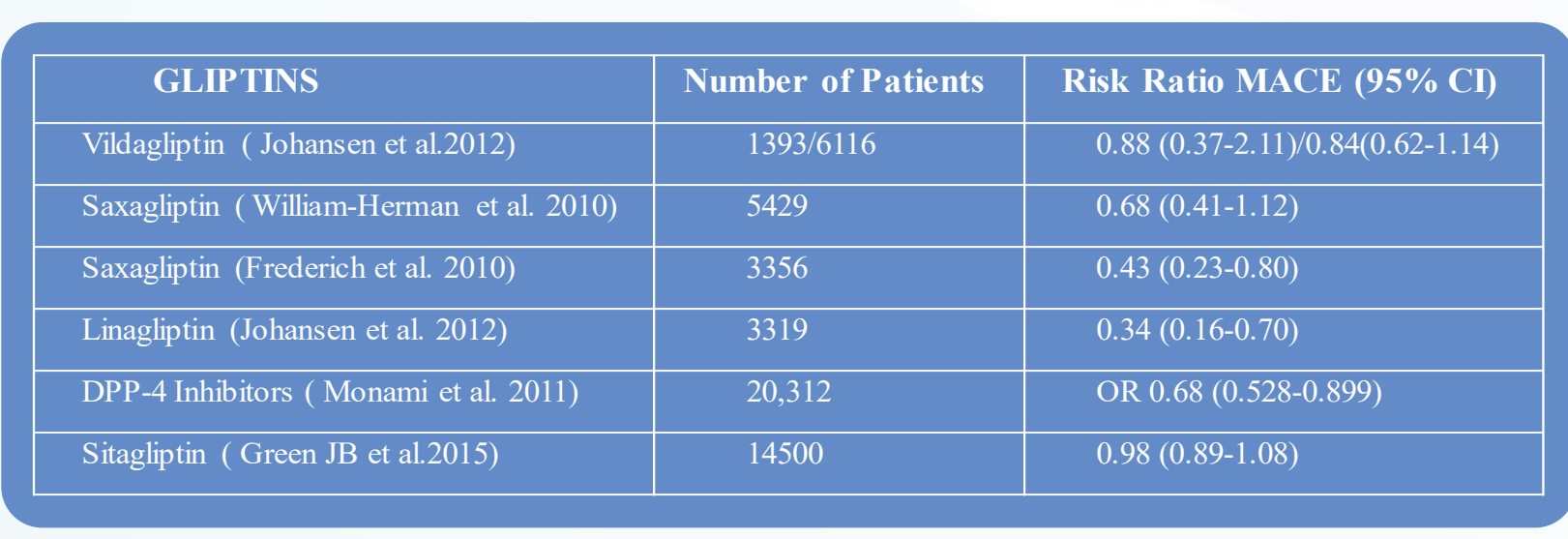

\begin{tabular}{|l|l|l|l|l|}
\hline Sitagliptin & Vildagliptin & Linagliptin & Saxagliptin & Alogliptin \\
\hline & 0.67 & -0.74 & -0.74 \\
\hline
\end{tabular}

\section{RESULTS}

Relevant studies were searched on Medline, PubMed, Embase and Cochrane Central CV effects of DPP-4 Inhibitors. The inclusion criteria for analysis included study duration of at least 12 weeks and enrolment of more than 30 participants. HbA reducered as major adverse cardiovascular events (MACE) in non-pregnant adults with T2DM. Type 1 diabetes mellitus patients were excluded from this review.

\section{Source: Stuart A. Ross. (2010). Incertin agents I type 2 diabetes.Canadian Family Physician, 56(7), pp. 639-648.}

\section{CONCIUSION}

